\title{
Medical Algorithm: Early Introduction of Food Allergens in High Risk Populations
}

\author{
Helen Fisher ${ }^{1}$, Gideon Lack $^{2}$, Graham Roberts ${ }^{3}$, Henry Bahnson ${ }^{4}$, and George Du Toit ${ }^{2}$ \\ ${ }^{1}$ King's College London, and Guy's and St. Thomas' NHS Foundation Trust \\ ${ }^{2}$ Department of Paediatric Allergy, Division of Asthma, Allergy and Lung Biology, King's \\ College London and Guy's and St. Thomas' NHS Foundation Trust, London \\ ${ }^{3}$ University of Southampton \\ ${ }^{4}$ Immune Tolerance Network
}

May 28, 2020

Medical Algorithm: Early Introduction of Food Allergens in High Risk Populations

Helen R Fisher, ${ }^{1,2}$ Gideon Lack, ${ }^{1,2,3}$ Graham Roberts, ${ }^{4,5,6}$ Henry T Bahnson, ${ }^{7}$ George Du Toit. ${ }^{1,2,3}$

${ }^{1}$ Paediatric Allergy Group, Department of Women and Children's Heath, School of Life Course Sciences, King's College London, London, United Kingdom

${ }^{2}$ Paediatric Allergy Group, Peter Gorer Department of Immunobiology, School of Immunology and Microbial Sciences, King's College London, London, United Kingdom.

${ }^{3}$ Children's Allergy Service, Guy's and St. Thomas' NHS Foundation Trust, London, United Kingdom.

${ }^{4}$ The David Hide Asthma and Allergy Research Centre, St Mary's Hospital, Newport, UK.

${ }^{5}$ NIHR Biomedical Research Centre, University Hospital Southampton NHS Foundation Trust, Southampton, UK.

${ }^{6}$ Faculty of Medicine, Clinical and Experimental Sciences, Human Development in Health Academic Units, University of Southampton, Southampton, UK.

${ }^{7}$ Immune Tolerance Network, Benaroya Research Institute, Seattle, Washington

Corresponding Authour:

Professor George Du Toit

Paediatric Allergy

Block B, South Wing

St Thomas' Hospital

London

SE1 7EH

Tel: 02071889784

Email: George.dutoit@gstt.nhs.uk

Word Count: 602 
Tables: 0

Figures: 1

Oral Tolerance Induction (OTI) is the only RCT-proven effective intervention for preventing childhood food allergy.(1) OTI to peanut is effective in a general population, with the greatest effect, $81 \%$ RRR, noted in the high-risk population.(2) OTI also reduced egg allergy in the general population.(1) Many governmental and allergy societies now recommend introducing peanut in infancy and some suggest other foods, such as well-cooked egg, are also introduced. Choosing which infants should undergo OTI, at what age, to which foods, and under which circumstances is critical for successful OTI prevention in populations where food allergy is a public health concern.

Infants with eczema are at increased risk of food allergy but infants from the general population are also at risk and contribute most cases at a population level. Risk of food sensitisation or food allergy increase with age; OTI is most likely to be successful when started in early infancy. Oral tolerance induction from 4 months of age, when completed using standard foods, is safe for nutrition, growth and general child health outcomes (3). Commencing multiple food OTI at 4 months of age, has no detrimental effect on established breastfeeding.(4) All children should adopt a diverse weaning diet, including allergenic foods such as wellcooked egg and peanut, as soon as weaning commences. High risk children should not delay weaning but start weaning and actively include peanut and well-cooked egg, as soon as developmentally ready; usually at about 4 months of age (Fig 1 ).

A $2 \mathrm{~g}$ /week dosing regime of peanut and well-cooked egg in early infancy is more effective in inducing oral tolerance than later introduction.(5) A lower dosing regime has not been shown to be effective in preventing allergy but, importantly, does not increase allergy risk above that of children who introduce allergenic foods in later infancy.(4) There are limited data regarding the efficacy of OTI to other allergenic foods, or the dose required.(1) All infants should aim to consume about $2 \mathrm{~g}$ of peanut protein and well-cooked egg per week; parents of high-risk infants should give these amounts more diligently. Given the benefit observed for peanut and egg, it is reasonable for all weaning infants to additionally incorporate $2 \mathrm{~g}$ of other common and nutritious food allergens; cow's milk (e.g. as yoghurt), wheat, fish and sesame.

Whether children should undergo allergy testing and/or have their first feed of peanut under medical supervision is contested. This cautious approach, potentially requiring large numbers of children to access specialist allergy care, must be balanced against the risks of severe allergic reaction, particularly as most allergic reactions occur on first oral exposure. RCTs of OTI using whole foods had no cases of anaphylaxis on first exposure $(4,6)$ although anaphylaxis has occurred to OTI using pasteurised whole egg powder.(7) Children with no personal food allergy risk factors do not require testing prior to, or medical supervision during, their first consumption of peanut or well-cooked egg. Children with moderate to severe eczema, or with an existing food allergy should undergo allergy testing +/- OFC at a specialist allergy centre(8), if doing so would not cause undue delay to OTI. It is likely that rapid access to allergy services will be further compromised as a consequence of the COVID-19 pandemic. It may however be that access to SpIgE is available through GP or paediatrician which, if [?]0.35KiU/L, will require referral for OFC. If negative $(<0.35 \mathrm{KiU} / \mathrm{L})$ the food may be introduced at home following precautionary measures for the first feed: child is well; parent is aware of the signs of IgE mediated reaction has, access to medical support if required and age-appropriate form of the food is given incrementally (Figure 1).

\section{References}

1. Ierodiakonou D, Garcia-Larsen V, Logan A, et al. Timing of allergenic food introduction to the infant diet and risk of allergic or autoimmune disease: A systematic review and meta-analysis. JAMA2016;316 (11):1181-1192.

2. Du Toit G, Roberts G, Sayre PH, Bahnson HT, Radulovic S, Santos AF, et al. Randomized trial of peanut consumption in infants at risk for peanut allergy. New England Journal of Medicine2015;372 (9):803-813.

3. Feeney M, Du Toit G, Roberts G, Sayre PH, Lawson K, Bahnson HT, et al. Impact of peanut consumption 
in the LEAP Study: Feasibility, growth, and nutrition. Journal of Allergy and Clinical Immunology ;138 (4):1108-1118.

4. Perkin MR, Logan K, Tseng A, Raji B, Ayis S, Peacock J, et al. Randomized Trial of Introduction of Allergenic Foods in Breast-Fed Infants. New England Journal of Medicine2016;374 (18):1733-1743.

5. Fisher HR, Du Toit G, Bahnson HT, Lack G. The challenges of preventing food allergy: Lessons learned from LEAP and EAT. Annals of Allergy, Asthma \& Immunology 2018;121 (3):313-319.

6. Du Toit G, Roberts G, Sayre PH, Bahnson HT, Radulovic S, Santos AF, et al. Randomized Trial of Peanut Consumption in Infants at Risk for Peanut Allergy. New England Journal of Medicine2015;372 (9):803-813.

7. Bellach J, Schwarz V, Ahrens B, Trendelenburg V, Aksünger Ö, Kalb B, et al. Randomized placebocontrolled trial of hen's egg consumption for primary prevention in infants. Journal of Allergy and Clinical Immunology ;139 (5):1591-1599.e1592.

8. Togias A, Cooper SF, Acebal ML, Assa'ad A, Baker JR, Beck LA, et al. Addendum guidelines for the prevention of peanut allergy in the United States. Pediatric Dermatology 2017;34 (1):5-12.

\section{Hosted file}

ALLERGY_algorithm_newsand views_FINAL.pptx available at https://authorea.com/users/327477/ articles/454986-medical-algorithm-early-introduction-of-food-allergens-in-high-riskpopulations 